\title{
Role of Protein Phosphatase 2A in Regulating the Visual Signaling in Drosophila
}

\author{
Ning Wang, ${ }^{1}$ Hung-Tat Leung, ${ }^{3}$ William L. Pak, ${ }^{3}$ Yonatan T. Carl, ${ }^{1}$ Brian E. Wadzinski, ${ }^{1}$ and Bih-Hwa Shieh ${ }^{1,2}$ \\ ${ }^{1}$ Department of Pharmacology, Center for Molecular Neuroscience, and ${ }^{2}$ Vision Research Center, Vanderbilt University, Nashville, Tennessee 37232 , and \\ ${ }^{3}$ Department of Biological Sciences, Purdue University, West Lafayette, Indiana 47907
}

Drosophila visual signaling, a G-protein-coupled phospholipase $\mathrm{C} \beta$ (PLC $\beta$ )-mediated mechanism, is regulated by eye-protein kinase C (PKC) that promotes light adaptation and fast deactivation, most likely via phosphorylation of inactivation no afterpotential D (INAD) and TRP (transient receptor potential). To reveal the critical phosphatases that dephosphorylate INAD, we used several biochemical analyses and identified protein phosphatase 2A (PP2A) as a candidate. Importantly, the catalytic subunit of PP2A, microtubule star (MTS), is copurified with INAD, and an elevated phosphorylation of INAD by eye-PKC was observed in three $m t s$ heterozygotes. To explore whether PP2A (MTS) regulates dephosphorylation of INAD by counteracting eye-PKC [INAC (inactivation no afterpotential C)] in vivo, we performed ERG recordings. We discovered that $i n a C^{P 209}$ was semidominant, because inaC ${ }^{P 209}$ heterozygotes displayed abnormal light adaptation and slow deactivation. Interestingly, the deactivation defect of $i n a C^{P 209}$ heterozygotes was rescued by the $m t s^{X E 2258}$ heterozygous background. In contrast, $m t s^{X E 2258}$ failed to modify the severe deactivation of norp $A^{P 16}$, indicating that MTS does not modulate NORPA (no receptor potential A) (PLC $\beta$ ). Together, our results strongly indicate that dephosphorylation of INAD is catalyzed by PP2A, and a reduction of PP2A can compensate for a partial loss of function in eye-PKC, restoring the fast deactivation kinetics in vivo. We thus propose that the fast deactivation of the visual response is modulated in part by the phosphorylation of INAD.

Key words: PP2A; PKC; deactivation; TRP; INAD; reversible phosphorylation

\section{Introduction}

Drosophila visual transduction is a G-protein-coupled signaling processes leading to the depolarization of photoreceptors by the opening the transient receptor potential (TRP) and TRP-like cation channels (Pak and Leung, 2003; Wang and Montell, 2007). In short, light activates rhodopsins that couple to Gq (Lee et al., 1990; Scott et al., 1995) resulting in activation of a phospholipase $\mathrm{C} \beta$ (PLC $\beta$ ), no receptor potential A (NORPA) (Bloomquist et al., 1988), which generates inositol trisphosphate and diacylglycerol (DAG). DAG may activate the TRP channel (Hardie, 2004) and is also a potent activator of protein kinase C (PKC). In Drosophila photoreceptors, DAG-activated eye-PKC is crucial for modulating deactivation, inactivation, and light adaptation of the visual signaling (Ranganathan et al., 1991; Hardie et al., 1993; Gu et al., 2005). At the molecular level, eye-PKC is tethered to a macromolecular complex by interacting with inactivation no afterpotential D (INAD), which is a scaffolding protein that organizes the formation of the protein complex by colocalizing NORPA, TRP, and eye-PKC (Huber et al., 1996b; Tsunoda et al.,

Received Oct. 5, 2007; revised Dec. 11, 2007; accepted Dec. 14, 2007.

This work was supported by National Eye Institute Grants EY09743 (B.-H.S.), EY00033 (W.L.P.), and EY08126 (to Vanderbilt University). We thank Sean Davey for the creation of the "ScanDenser" program. We thank Amy Ham and Jade Johnston in the Vanderbilt Proteomics Laboratory for the LC-MS/MS analysis of the microcystin pull-down. We thank Mingya Liu, Qin-Xia Chen, and Li Peng for excellent technical support.

Correspondence should be addressed to Dr. Bih-Hwa Shieh, Department of Pharmacology, 402 Robinson Research Building, Vanderbilt University Medical Center, Nashville, TN 37232-6600. E-mail: bih-hwa.shieh@ vanderbilt.edu.

DOI:10.1523/JNEUROSCI.5134-07.2008

Copyright $\odot 2008$ Society for Neuroscience $\quad$ 0270-6474/08/281444-08\$15.00/0
1997; Adamski et al., 1998; Li and Montell, 2000). Eye-PKC has been shown to phosphorylate INAD and TRP (Huber et al., 1996a, 1998; Liu et al., 2000; Popescu et al., 2006). Recently, we demonstrated that eye-PKC phosphorylates TRP at Ser ${ }^{982}$ in vivo, and a loss of phosphorylation in TRP leads to slow deactivation of the visual response (Popescu et al., 2006). To terminate the effect of eye-PKC, protein phosphatases are required for orchestrating a timely dephosphorylation, and ensure returning of INAD and TRP to their prephosphorylated states for subsequent regulation by eye-PKC.

Reversible phosphorylation plays an important role in modulating an array of cellular processes. Many protein kinases have been identified and their regulation characterized. However, less is known regarding the identity of critical protein phosphatases. Unlike protein kinases, protein phosphatases are more limited in number and thus are likely to have broad substrate specificity. Protein phosphatases that dephosphorylate phospho-serine/ threonine residues are subdivided into four major classes, protein phosphatase 1 ( $\mathrm{PP} 1)$, protein phosphatase $2 \mathrm{~A}$ ( $\mathrm{PP} 2 \mathrm{~A})$, protein phosphatase $2 \mathrm{~B}$, and protein phosphatase $2 \mathrm{C}$, based on their sensitivity to inhibitors and requirement for divalent cations (Barford, 1996). Protein phosphatase $2 \mathrm{~B}$ and protein phosphatase $2 \mathrm{C}$ exhibit a strict requirement for $\mathrm{Ca}^{2+}$ and $\mathrm{Mg}^{2+}$, respectively, whereas PP1 and PP2A do not. Moreover, both PP1 and PP2A are sensitive to okadaic acid and microcystin with different sensitivity (Barford, 1996). PP2A constitutes the majority of serine/threonine phosphatase activity in the cell. The predominant form of PP2A is a heterotrimeric complex consisting of catalytic (C), 
structural (A), and variable (B) subunits (Lechward et al., 2001). The $\mathrm{C}$ subunit of PP2A interacts constitutively with the A subunit, which acts as a scaffolding protein for binding the $\mathrm{C}$ subunit and variable B subunit. The B subunit plays a role in targeting PP2A holoenzymes to specific subcellular compartments and therefore is critical for regulating substrate specificity (Lechward et al., 2001).

Here, we present evidences that support a critical role of PP2A in Drosophila visual transduction by regulating the dephosphorylation of INAD. We used biochemical analyses including protein purification and inhibitor studies, and demonstrated that PP2A catalyzed the dephosphorylation of INAD. The $\mathrm{C}$ subunit of PP2A corresponds to the microtubule star $(m t s)$ gene product in Drosophila (Snaith et al., 1996). By electroretinogram (ERG) recording, we discover that $m t s^{X E 2258}$ is a recessive mutation, whereas inactivation no afterpotential C $\left(\right.$ ina $C^{P 209}$ ) is haploinsufficient, because ina $C^{P 209}$ heterozygotes display abnormal light adaptation and slow deactivation kinetics. Interestingly, only the deactivation defect of the ina $C^{P 209}$ heterozygotes was rescued in the $m t s^{X E 2258}$ heterozygous background. However, $m t s^{X E 2258}$ heterozygotes failed to restore the abnormal deactivation of nor$p A^{P 16}$, indicating that the function of NORPA is not regulated by MTS to modulate deactivation kinetics. Based on the findings, we conclude that MTS regulates multiple substrates in the visual cascade including INAD. A reduction of MTS can compensate for a partial loss of function in eye-PKC, leading to the rescue of the slow deactivation of the visual signaling in ina $C^{P 209}$ heterozygotes. We hypothesize that phosphorylation of INAD plays an important role in visual transduction, mediating fast deactivation kinetics.

\section{Materials and Methods}

In vitro phosphatase assays. To monitor dephosphorylation of INAD, phosphorylated INAD ${ }^{1-363}$, which was prepared by in vitro kinase reactions using a recombinant $\mathrm{PKC} \alpha$ (Calbiochem, La Jolla, CA), was incubated with fly extracts, eluates from various chromatographic steps or purified enzymes for $30 \mathrm{~min}$ at $30^{\circ} \mathrm{C}$ to monitor dephosphorylation. The reactions were terminated on the addition of trichloroacetic acid and analyzed by SDS-PAGE. The amount of phosphorylated INAD was detected by liquid scintillation counting or autoradiography. The A/C dimer of human PP2A and the rabbit PP1c (skeletal muscle) were purchased from Upstate Biotechnology (Lake Placid, NY).

Preparation of fly head extracts, and Western blotting. Fly heads were extracted with extraction buffer (50 mm Tris- $\mathrm{HCl}, \mathrm{pH} 8.0,150 \mathrm{~mm} \mathrm{NaCl}$, $1 \%$ Triton X-100, and a mixture of protease inhibitors) as detailed previously (Popescu et al., 2006). For quantitative Western blotting, fluorophore-conjugated secondary antibodies (Alexa Fluor 680 goat anti-rabbit IgG; Invitrogen, Carlsbad, CA) were used and the signals were visualized and quantified by the Odyssey Infrared Imaging system (LICOR, Lincoln, NE). Polyclonal antibodies against INAD (Shieh and Niemeyer, 1995), eye-PKC (Adamski et al., 1998), and TRP (Shieh and Zhu, 1996) were obtained as described previously. Monoclonal anti-PP2Ac $\alpha$ and anti-PP1 were obtained from BD Biosciences (San Jose, CA).

Immunoprecipitation and immunocomplex kinase assay. Fly head extracts (300 $\mu \mathrm{g}$ total protein) were incubated with anti-INAD antibodies, and the INAD immunocomplexes were recovered by protein A agarose beads (Pierce, Rockford, IL). After three washes with the buffer, the immunocomplexes were analyzed by SDS-PAGE and Western blotting, or proceeded with an in vitro kinase reaction by incubating with $50 \mu \mathrm{l}$ of kinase reaction buffer (50 mM Tris- $\mathrm{HCl}, \mathrm{pH} 8.0,10 \mathrm{mM} \mathrm{MgCl}_{2}, 5 \mathrm{~mm}$ 2-mercaptoethanol, 0.1 mM DTT, 0.4 mM EGTA, $0.7 \mathrm{~mm} \mathrm{CaCl}_{2}$ ) containing $3 \mu \mathrm{Ci}$ of carrier-free $\left[\gamma_{-}{ }^{32} \mathrm{P}\right] \mathrm{ATP}$ (PerkinElmer, Boston, MA) and phorbol myristate acetate $(1 \mu \mathrm{M})$ at $30^{\circ} \mathrm{C}$ for $30 \mathrm{~min}$. The kinase reaction was terminated on the addition of SDS-PAGE sample buffer, analyzed by SDS-PAGE, followed by PhosphorImager analysis (GE Healthcare Life Sciences, Piscataway, NJ) or autoradiography.
Partial purification of INAD phosphatases by FPLC. Drosophila heads (5-10 g) were homogenized using a solubilization buffer $(50 \mathrm{~mm}$ Tris$\mathrm{HCl}, \mathrm{pH} 7.8,150 \mathrm{~mm} \mathrm{NaCl}, 1 \mathrm{~mm}$ EDTA, plus a mixture of protease inhibitors). The supernatant was collected after centrifugation and brought to $20 \%$ ammonium sulfate saturation. The desired phosphatase activity was found enriched in proteins precipitated between 20 and $40 \%$ saturation, which were collected and fractionated over a Phenyl Sepharose (GE Healthcare Life Sciences) column. Briefly, the precipitated proteins were resuspended in buffer A (50 mM Tris- $\mathrm{HCl}, \mathrm{pH} 7.8,0.1 \mathrm{~mm}$ EDTA, $10 \%$ glycerol, $2 \mathrm{~mm} \mathrm{MgCl}_{2}$ plus protease inhibitors), and solid ammonium sulfate was added to a final concentration of $1.0 \mathrm{M}$. The mixture was incubated on ice for $30 \mathrm{~min}$ and centrifuged. The supernatant was loaded onto a Phenyl Sepharose column $(30 \mathrm{ml})$ preequilibrated with buffer A containing $1 \mathrm{~m}$ ammonium sulfate, and proteins was eluted over a linear gradient of ammonium sulfate $(1-0 \mathrm{M})$. The phosphatase activity that dephosphorylates INAD was eluted at $0 \mathrm{M}$ ammonium sulfate. Fractions containing the desired phosphatase from five successive runs of Phenyl Sepharose column were pooled and loaded onto Q Sepharose (GE Healthcare Life Sciences) column (12 ml). Proteins were eluted using a linear gradient of $\mathrm{NaCl}(0-600 \mathrm{~mm})$. The INAD phosphatase usually was eluted at $300 \mathrm{~mm} \mathrm{NaCl}$. Fractions enriched in the phosphatase activity were also analyzed by SDS-PAGE, followed by Western blotting. Alternatively, microcystin agarose (Millipore, Billerica, MA) was used to enrich for members of the PP1 and PP2A family, which were subsequently analyzed by mass spectrometry.

Liquid chromatography and tandem mass spectrometry. Proteins bound to microcystin beads were digested directly off of the beads with trypsin, and the tryptic peptides were separated by HPLC. The liquid chromatography-tandem mass spectrometry (LC-MS/MS) analysis was performed on a ThermoFinnigan LTQ linear ion trap mass spectrometer equipped with a ThermoFinnigan Surveyor autosampler and LC pump, NanoSpray source (Thermo Electron, San Jose, CA), and Xcalibur 1.4 instrument control and data analysis software. LC/MS was performed by the Proteomics Laboratory/Mass Spectrometry Research Center at Vanderbilt.

Database searching and data analysis. The "ScanDenser" algorithm read tandem mass spectra stored as centroided peak lists from Thermo RAW files and transcoded them to DTA files. Proteins were identified using the SEQUEST algorithm via the SEQUEST Browser software in the Bioworks 3.1 package (Thermo Electron) (Yates et al., 1995) on a desktop computer. The data were searched against the Drosophilia subset of the NCBI database.

Fly stocks. Fly stocks were maintained at $25^{\circ} \mathrm{C}$ in a $12 \mathrm{~h}$ dark/light cycle. The three $m t s$ alleles $\left(m t s^{02496}, m t s^{k 12502}\right.$, and $\left.m t s^{X E 2258}\right), r d g C^{306}$, and $g l a^{3}$ flies were obtained from Bloomington Stock Center (Bloomington, IN). The original $m t s^{X E 2258}$ stock was crossed with w; CyO/Sco to remove the P-element containing a dominant Ras transgene.

Recombination between $\mathrm{mts}^{\mathrm{XE} 2258}$ and $\mathrm{inaC}^{\mathrm{P} 209}$. The white-eyed $m t s^{X E 2258}$, ina $C^{P 209} / \mathrm{CyO}$ line was generated with a scheme involving five crosses. First, we obtained virgin females of $m t s^{X E 2258} / i n a C^{P 209}$, which were crossed with males of $w$; $S c o / C y O$ and the individual male progeny with possible recombination of $m t s^{X E 2258}$ and ina $C^{P 209}$ was collected. Individual male was mated female $w$; $S c o / C y O$ to generate a line, and 12 independent lines were established by saving potential $m t^{X E 2258}$, in$a C^{P 209} / C y O$ for a stock. These 12 potential double mutant lines were examined for presence of the ina $C^{p 209}$ and $m t s^{X E 2258}$ mutations: the presence of $m t^{X E 2258}$ was confirmed for the inability of the line to generate homozygotes as only heterozygous flies (over $\mathrm{CyO}$ balancer chromosome) can survive to adult. The presence of ina $C^{P 209}$ was confirmed by a backcross with ina $C^{P 209}$, in which $\mathrm{F}_{1}$ of the following genotype, $m t^{X E 2258}$, ina $C^{P 209} /$ ina $^{P 209}$, was selected and analyzed for the absence of eye-PKC by Western blotting.

ERG recordings. ERGs were performed on 3- to 5-d-old flies as described previously (Larrivee et al., 1981). A $300 \mathrm{~W}$ halogen lamp (Osram, Danvers, MA) was used for light stimuli. The unattenuated intensity at the level of the fly was $810 \mu \mathrm{W} / \mathrm{cm}^{2}$. A $15 \mathrm{~W}$ tungsten lamp was used to provide background light. The unattenuated light intensity of the background light at the level of the fly was $8 \mu \mathrm{W} / \mathrm{cm}^{2}$, which is $\sim 2 \log$ units dimmer than the unattenuated light source used for light stimulus. 
Kodak neutral filters were used to achieve the desired light intensity for the light stimulus and the background lights. At each stimulus, the flies were first dark-adapted for $3 \mathrm{~min}$ and then given a white light stimulus (5 or $1 \mathrm{~s}$ ). When background lights were used, the flies were dark adapted for $3 \mathrm{~min}$, and then background light of a particular intensity was turned on for $30 \mathrm{~s}$ before a $5 \mathrm{~s}$ light stimulus was given to the eye. After the light stimulus, the background light was then turned off. To ensure photoconversion of metarhodopsin back to rhodopsin, an orange light stimulus was given after each stimulus. All recordings were made at $25^{\circ} \mathrm{C}$. Signals were sampled at $2 \mathrm{kHz}$ with an analog-to-digital converter (Digidata $1200 \mathrm{~A}$ ), and the data were acquired and analyzed in a computer with Axoscope (Molecular Devices, Sunnyvale, CA).

\section{Results}

Dephosphorylation of INAD is mediated by ubiquitously expressed protein phosphatases

Previously, we had shown that protein phosphatases in the cytosolic extracts of wild-type fly heads could dephosphorylate INAD (Liu et al., 2000), and the critical protein phosphatases appeared to be enriched in photoreceptors. Because most of protein phosphatases seem to be ubiquitously expressed, we reinvestigated whether dephosphorylation of INAD is indeed mediated by protein phosphatases specifically expressed in photoreceptors by examining extracts prepared from glass flies that lack photoreceptors (Moses et al., 1989).

To better detect protein phosphatase activity, we generated a highly phosphorylated INAD as substrates. Experimentally, affinity-purified GST fusion proteins containing an INAD fragment spanning residues $1-363$ (INAD ${ }^{1-363}$ ) was phosphorylated by a recombinant $\mathrm{PKC} \alpha$, which appears to display similar substrate specificity as eye-PKC in vitro (Popescu et al., 2006). Indeed, INAD ${ }^{1-363}$ became intensely phosphorylated by $\operatorname{PKC} \alpha$, when compared with phosphorylation of fusion proteins containing the remaining INAD sequence (i.e., INAD ${ }^{364-674}$ ) (L. Peng and B.-H. Shieh, unpublished observations). For in vitro dephosphorylation assays, phosphorylated INAD ${ }^{1-363}$ was incubated with cytosolic extracts from either wild-type or glass flies. We found that glass extracts dephosphorylated INAD ${ }^{1-363}$ (Fig. $1 A$ ) similar to wild-type extracts, indicating that dephosphorylation of INAD is not regulated by photoreceptor-specific protein phosphatases. We also investigated whether retinal degeneration $\mathrm{C}$ (RDGC) was involved in the dephosphorylation of INAD, and found that $r d g C^{306}$ extracts also were able to dephosphorylate INAD $^{1-363}$ (Fig. 1 A). This finding indicates that RDGC, a protein phosphatase of the PP7 family (Steele et al., 1992) involved in the dephosphorylation of rhodopsin, does not play a role in the dephosphorylation of INAD. Together, it appears that dephosphorylation of INAD is regulated by ubiquitously expressed protein phosphatases. The discrepancy with our previous result may be partly contributed by a lower sensitivity of our previous dephosphorylation studies because the phosphorylation of INAD was less uniform in the immunocomplex kinase reactions affecting the sensitivity of the subsequent dephosphorylation assays.

\section{Okadaic acid inhibits dephosphorylation of INAD}

Previous work from our laboratory showed that both microcystin and okadaic acid suppressed the dephosphorylation of INAD and TRP in fly extracts (Liu et al., 2000). Here, we reinvestigated the effect of okadaic acid using phosphorylated INAD ${ }^{1-363}$. As shown in Figure $1 B$, okadaic acid displayed a dose-dependent inhibition leading to increased levels of phosphorylated INAD. It has been reported that okadaic acid suppresses purified PP2A with an $\mathrm{IC}_{50}$ of $0.1 \mathrm{nM}$, whereas it inhibits purified PP1 with an $\mathrm{IC}_{50}$ of $10 \mathrm{~nm}$ (Barford, 1996). We estimated the $\mathrm{EC}_{50}$ of okadaic acid in pre-

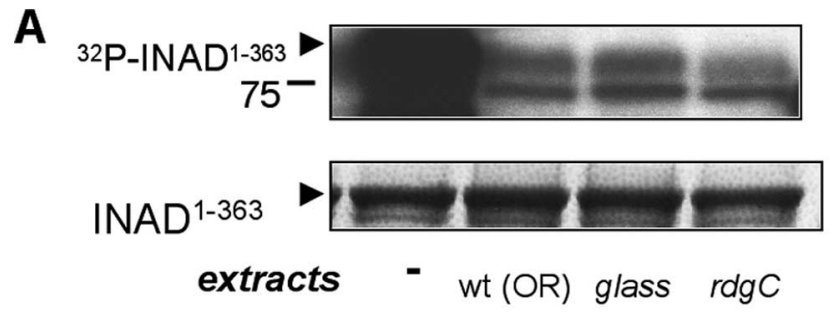

\section{B}

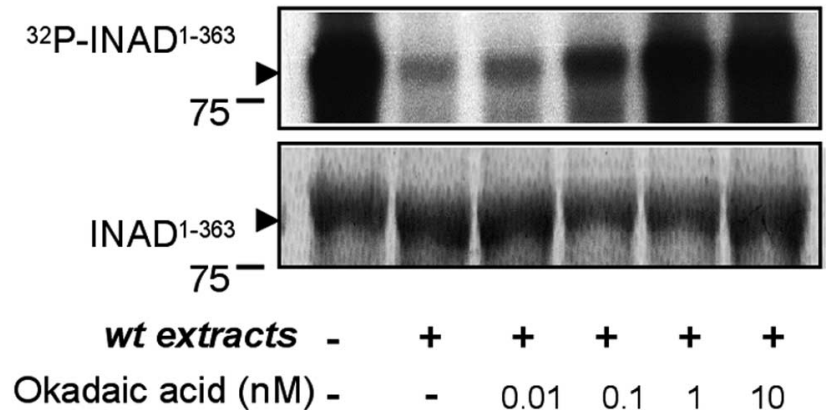

Figure 1. Protein phosphatases involved in dephosphorylation of INAD. A, Dephosphorylation of INAD is regulated by ubiquitously expressed protein phosphatases. A fixed amount of phosphorylated INAD ${ }^{1-363}$ obtained by in vitro PKC $\alpha$ phosphorylation was incubated with cytosolic extracts from fly heads. The protein phosphatase activity in the extracts (protein concentrations, $3 \mathrm{mg} / \mathrm{ml})$ prepared from wild-type, glass ( $\left.g / a^{3}\right)$, and $r d g C\left(r d g c^{306}\right)$ were compared. Top, Autoradiogram; bottom, the Coomassie staining. $\boldsymbol{B}$, Dephosphorylation of INAD is inhibited by okadaic acid. The effect of okadaic acid (0.01-10 nm) to suppress the phosphatase activity in wild-type extracts was investigated. Levels of phosphorylated INAD ${ }^{1-363}$ are shown on the top. The bottom panel shows the amount of INAD ${ }^{1-363}$ by the Coomassie staining.

venting the dephosphorylation of INAD to be $\sim 0.3 \mathrm{nM}$, strongly supporting our hypothesis that a member of the PP2A family is responsible for the dephosphorylation of INAD.

\section{PP2A is a candidate protein phosphatase for INAD: evidence from protein purification}

To identify the candidate protein phosphatases involved in the dephosphorylation of INAD, we took a biochemical approach by isolating the phosphatase activities from the cytosolic extracts using two consecutive chromatographic steps that were used successfully to purify members of the PP1/2A family (Kloeker and Wadzinski, 1999). Briefly, cytosolic extracts of fly heads were subjected to ammonium sulfate precipitation (20-40\%), and the protein precipitates were subsequently dissolved and fractionated on a Phenyl Sepharose column by a linear gradient of ammonium sulfate (1-0 M) (Fig. 2A). Positive fractions (eluted at 0 $\mathrm{M}$ ammonium sulfate) were recovered, concentrated, and purified on a Q Sepharose column over a gradient of $\mathrm{NaCl}(0-600$ $\mathrm{mm})$. The desired phosphatase activities were eluted at $\sim 300 \mathrm{~mm}$ $\mathrm{NaCl}$ (Fig. 2B).

All fractions were also analyzed by Western blotting using commercially available antibodies against the $\mathrm{C}$ subunit of PP2A, PP2Ac. We found that anti-PP2Ac antibodies recognized a protein of $\sim 35 \mathrm{kDa}$ similar to the estimated molecular mass of Drosophila PP2Ac or MTS in all positive fractions (Fig. 2C). We also tested for the presence of the C subunit of PP1, PP1c, using several commercially available antibodies. However, none of these antibodies seemed to recognize Drosophila PP1c in either cytosolic extracts or enriched extracts after ammonium sulfate precipitation (data not shown).

To further confirm the copurification of PP2A with the ex- 

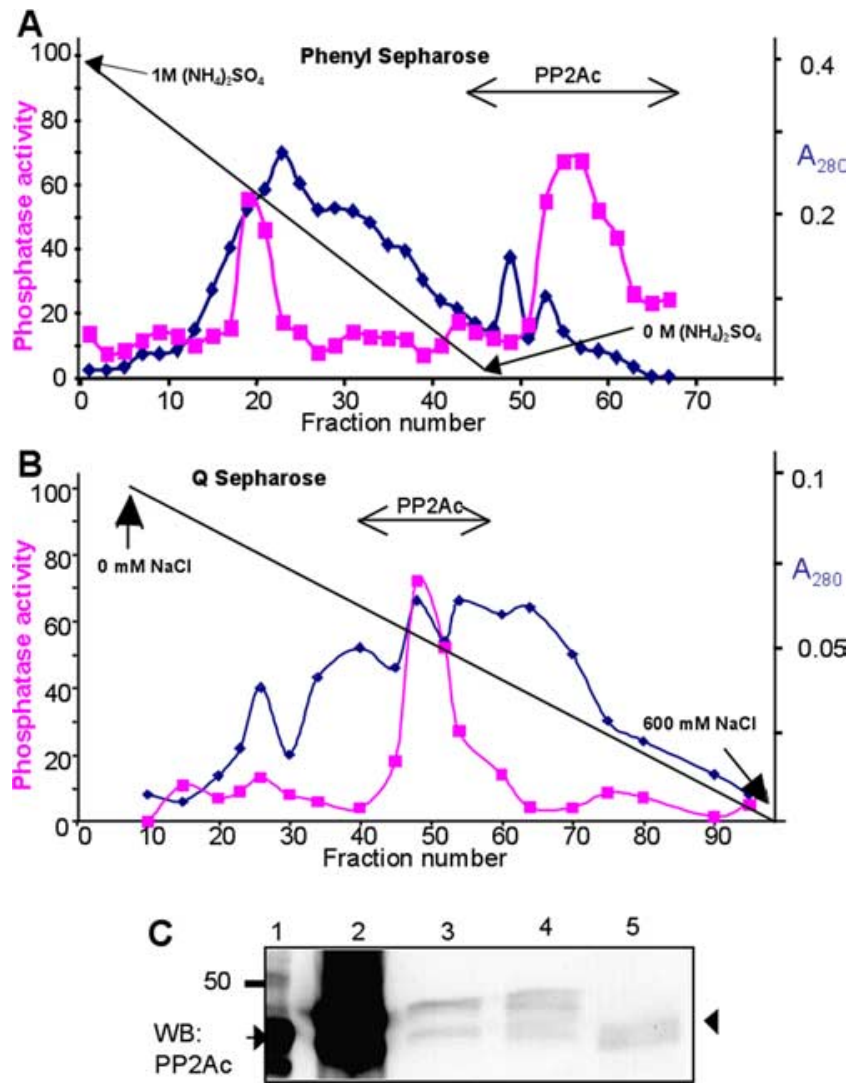

Figure 2. Partial purification of the phosphatase that dephosphorylates INAD. A, B, Enrichment of the phosphatase activity by Phenyl Sepharose chromatography after ammonium sulfate precipitation $(\boldsymbol{A})$, and the subsequent purification over $Q$ Sepharose $(\boldsymbol{B})$. Shown are elution profiles of total proteins based on absorption at $280 \mathrm{~nm}$ (blue), the phosphatase activity that dephosphorylated INAD (magenta), and the PP2A immunoreactivity (PP2Ac). C, Copurification of PP2AC in positive fractions that dephosphorylated INAD. Shown is a Western blot using anti-PP2Ac antibodies. Lane 1, Cytosolic extracts; lane 2, ammonium sulfate precipitation; lane 3, Phenyl Sepharose eluates; lane 4, Q Sepharose eluates; lane 5, microcystin agarose eluates. The $50 \mathrm{kDa}$ protein standard is indicated on the left.

pected phosphatase activity for INAD, we used a proteomic approach. Positive fractions eluted from Q Sepharose were enriched for PP2Ac and PP1c using microcystin agarose beads, which binds specifically to the C subunit of both PP2A and PP1. Bound $\mathrm{C}$ subunits were washed and subsequently analyzed by mass spectrometry. We detected three nonoverlapping peptides (KYGNANVWKY, RRGEPHVTRR, and KFSFLQFDPAPRR) correspond to Drosophila PP2Ac in the microcystin pull-down, indicating that PP2Ac is copurified with the phosphatase activity that dephosphorylates INAD. Together, these findings strongly support that PP2A is involved in the dephosphorylation of INAD in vitro.

\section{Dephosphorylation of INAD by purified PP2Ac in vitro}

To further investigate whether INAD is a substrate of PP2A, we tested the ability of purified PP2A to dephosphorylate INAD in vitro. We show that purified human $\mathrm{A} / \mathrm{C}$ dimer of $\mathrm{PP} 2 \mathrm{~A}$ in which the C subunit shares $94.5 \%$ sequence identities with Drosophila MTS, displays a dose-dependent activity to dephosphorylate INAD (Fig. 3). In contrast, purified rabbit PP1c, which displays $>90 \%$ sequence identities with the fly homologues, exhibits no detectable activity toward phosphorylated INAD (Fig. 3). These results further confirm the critical role of PP2A to catalyze the dephosphorylation of INAD.
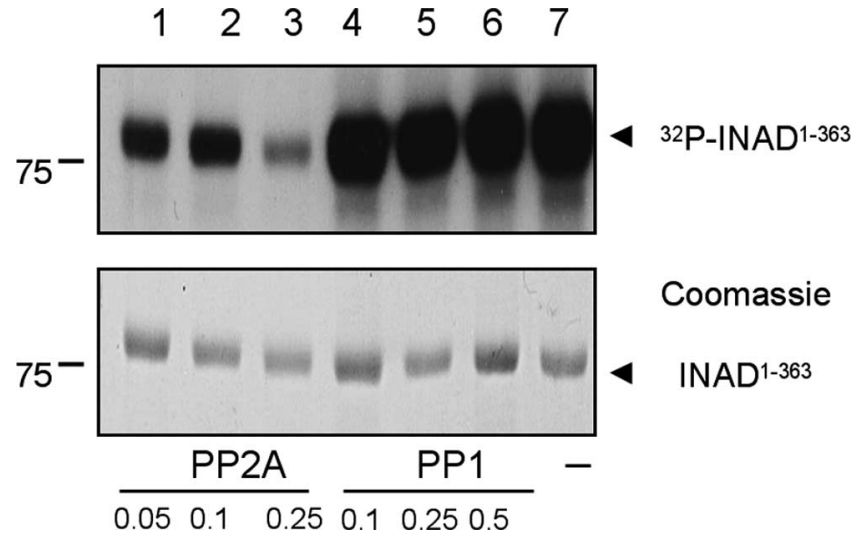

Figure 3. Dephosphorylation of INAD by purified human PP2Ac but not by rabbit PP1C. Phosphorylated INAD ${ }^{1-363}$ was incubated with indicated amount (in units) of purified human A/C dimer of PP2A and the ( subunit of rabbit PP1 (skeletal muscle) for 30 min and analyzed by SDS-PAGE. Lane 7 contains phosphorylated INAD ${ }^{1-363}$ alone. Phosphorylated INAD was detected by autoradiogram (top), and total INAD ${ }^{1-363}$ content was revealed by the Coomassie staining (bottom). Protein standards are indicated on the left.

MTS associates with the INAD complex and the $m t s$ heterozygotes display increased phosphorylation of INAD by in vitro kinase assays

If $\mathrm{PP} 2 \mathrm{~A}$ regulates dephosphorylation of INAD either directly or indirectly, a reduction of PP2A may lead to an increased phosphorylation of INAD by eye-PKC. Therefore, we monitored phosphorylation of INAD using immunocomplex kinase assays in three $m$ ts heterozygotes, because the corresponding homozygotes cannot be obtained for the analysis because of embryonic lethality (Wassarman et al., 1996). Experimentally, anti-INAD antibodies were used to isolate the INAD complex from fly head extracts, the immunocomplexes were recovered by protein A agarose beads, and phosphorylation of INAD by eye-PKC was monitored on the addition of $\mathrm{Ca}^{2+}$, phorbol ester, and radioactive ATP. As shown in Figure 4, phosphorylation of INAD was significantly increased compared with that of TRP in these $m t s$ alleles including $m t s^{02496}, m t s^{k 12502}$, and $m t s^{X E 2258}$ (Fig. $4 B$ ). This enhanced phosphorylation of INAD could be attributable to either increased eye-PKC activity and/or decreased dephosphorylation of INAD in the $m t s$ mutants. The latter is more likely because $m t s$ heterozygotes have a reduced level of PP2A (Wassarman et al., 1996).

The increased phosphorylation of INAD by the immunocomplex kinase assays using $m$ ts extracts suggests that PP2A is present in the INAD complex to modulate dephosphorylation of INAD. To investigate whether PP2A associates with the INAD complex, we performed coimmunoprecipitation assays using wild-type head extracts (Fig. 4C). Indeed, we found that anti-INAD antibodies copurified PP2A (MTS), whereas preimmune serum did not. This finding strongly indicates that PP2A interacts with INAD, and may represent an integral component of the INAD complex.

$\boldsymbol{m} t \boldsymbol{s}^{\mathrm{XE2258}}$ is a recessive visual mutation as heterozygotes of $\boldsymbol{m t s}^{\mathrm{XE2258}}$ exhibit wild-type ERG responses

To gain insight into the role of PP2A (MTS) in the visual signaling, we characterized the visual electrophysiology of $m t s^{\text {XE2258 }}$ heterozygotes, which display the most enhanced phosphorylation of INAD among the three alleles we tested. $m t^{\text {XE2258 }}$ also has been examined in studies related to regulation of eye development (Wassarman et al., 1996), circadian rhythm (Sathyanaray- 


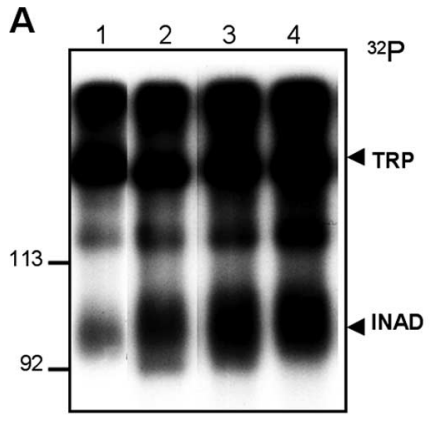

B

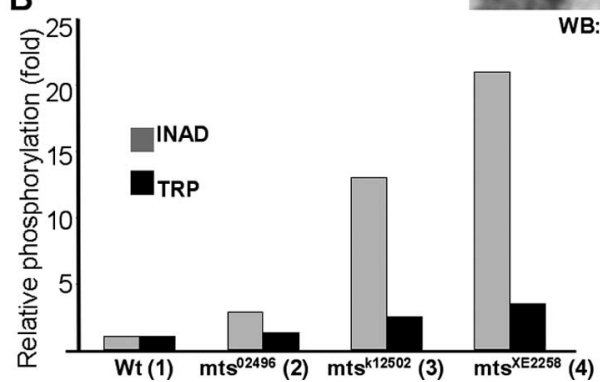

Figure 4. MTS is present in the INAD complex and modulates the level of phosphorylated INAD. Phosphorylation of INAD by eye-PKC was performed by immunocomplex kinase assays in which anti-INAD antibodies were used to isolate the INAD complex from extracts prepared from wild-type (lane 1) and three $m t s$ alleles, $m t^{02496}$ (lane 2), $m t^{k 12502}$ (lane 3), and $m t^{{ }^{X E 2258}}$ (lane 4). Shown is a representative autoradiogram depicting phosphorylation of INAD and TRP (A). Protein standards are indicated on the left. The relative level of phosphorylation, compared with that of wild-type extracts, was quantitated and shown in a histogram (B). C, MTS is coimmunoprecipitated by anti-INAD antibodies in wild-type head extracts. Shown is a Western analysis of the immunoprecipitation assays using anti-INAD antibodies or preimmune sera (lgG). AntiINAD antibodies copurified MTS (bottom), as well as NORPA (top) and INAD.

anan et al., 2004), and calcium/calmodulin-dependent protein kinase II (CaMKII) (Lu et al., 2003). Molecular characterization of $m t s^{X E 2258}$ reveals a $16 \mathrm{nt}$ deletion spanning its translation start site (Wassarman et al., 1996). By Western blotting, the MTS level in a single fly head of $m t s^{X E 2258}$ heterozygotes was estimated to be $74 \%(74 \pm 5 \%$, SEM; $n=3)$ of wild-type. Importantly, $m t s^{\text {XE2258 }}$ is white-eyed and thus better suited for electrophysiological analysis because white-eyed is sensitive to a wider range of light stimulus than red-eyed fly.

We performed ERG, an extracellular recording of the compound eye, and discovered that $m t^{X E 2258}$ heterozygotes ( $m t s$ / CyO) exhibited ERG waveform similar to that of wild type (wt or $+/ \mathrm{CyO}$ ) (Fig. 5A). We also compared the peak amplitude, half deactivation time (Fig. $5 G$ ), and the relative inactivation (Fig. $5 H$ ) of the ERG response, but failed to find significant differences with those of wild type. Moreover, $m t^{X E 2258}$ heterozygotes display no light adaptation defects (Fig. $6 \mathrm{~B}$ ). These findings indicate that a partial loss of functions of PP2A does not significantly modify Drosophila visual electrophysiology in $m t s^{\text {XE2258 }}$ heterozygotes.

\section{InaC is haploinsufficient because ina $C^{P 209}$ heterozygotes} display slow deactivation of the visual signaling Although $m t s^{\text {XE2258 }}$ did not display any electrophysiological defects, it is still possible that $m t s^{X E 2258}$ may modify a partial loss of function of eye-PKC that phosphorylates INAD. Therefore, we characterized heterozygotes of ina $C^{P 209}$. The ina $C^{P 209}$ allele is a null allele of eye-PKC because it contains a nonsense mutation at $\operatorname{Trp}^{93}$ (Smith et al., 1991). ina $C^{P 209}$ was originally classified as a recessive mutation because homozygotes have been shown to display multiple defects in light adaptation, inactivation, and deactivation. Unexpectedly, we found that ina $C^{P 209}$ heterozygotes (inaC/CyO) displayed ERG with slow deactivation kinetics, whereas ina $C^{P 209}$ homozygotes exhibited a more severe deactivation defect (inaC/inaC) (Fig. 5C), when stimulated with a 5 s light pulse of a bright light. In contrast, only ina $C^{P 209}$ homozygotes display response inactivation as the receptor potential rapidly declines during the light pulse. Our results are consistent with the role of eye-PKC to modulate response inactivation and fast deactivation. Importantly, it appears that the deactivation defect is in a reciprocal relationship to the gene dosage for eye-PKC in the ina $C^{P 209}$ mutants.

To quantitatively compare the deactivation and inactivation defects in these flies, we calculated "inactivation" as the percentage reduction of the peak amplitude during the light pulse [ 1 $s / p) \times 100 \%$; $p$, initial peak amplitude; $s$, peak amplitude at the end of $5 \mathrm{~s}$ light pulse] (for a graphic depiction, see Fig. 5D). As a parameter for the deactivation kinetics, we also measured half deactivation time as the time to reach $50 \%$ recovery after the light pulse (the elapsed time from $y$ to $z$ ) (Fig. 5D). Indeed, our results show that the half deactivation time of ina $C^{P 209}$ heterozygotes is twice as long as that of wild type, whereas the half deactivation time of inaChomozygotes is about three times longer than that of ina $C^{P 209}$ heterozygotes (Fig. 5G). Additionally, ina $C^{P 209}$ homozygotes, but not the heterozygotes, exhibit a greater degree of response inactivation under this stimulation condition (Fig. $5 \mathrm{H}$ ). Based on these findings, we conclude that inaC is haploinsufficient because two copies of the ina $C$ gene are required for a wildtype deactivation of the visual response.

\section{The deactivation defect of the ina $C^{P 209}$ heterozygotes can be rescued when $\mathrm{PP} 2 \mathrm{~A}$ is reduced}

The slow deactivation that we discovered in the ina $C^{P 209}$ heterozygotes can be caused by a reduced phosphorylation of the eye-PKC substrates, whereas we have shown that a reduction of PP2A leads to an increased level of phosphorylated INAD in the $m$ ts heterozygotes (Fig. 4). To explore the relationship between PP2A (MTS) and eye-PKC in vivo, we investigated whether $m t s^{X E 2258}$ rescues the abnormal deactivation of the ina $C^{P 209}$ heterozygotes. We generated and characterized $m t s^{X E 2258}$, ina $C^{P 209}$ double heterozygotes ( $m t s$, inaC/CyO) by ERG. Surprisingly, we found that double heterozygotes display an ERG waveform similar to that of wild type with normal deactivation kinetics (Fig. $5 E$ ), and comparable half deactivation times (Fig. $5 G$ ). This result strongly supports the notion that a reduction of the PP2A activity rescues the deactivation defect of ina $C^{P 209}$ heterozygotes. Based on these findings, we conclude that a reduction of PP2A compensates for a partial loss of function in eye-PKC to restore phosphorylation of INAD leading to normal deactivation kinetics of the visual response.

In contrast, we show that $m t s^{X E 2258}$ fails to rescue the response inactivation defect of the ina $C^{P 209}$ homozygotes (Fig. $5 F, H$ ). It is of great interest to note that, despite showing abnormal response inactivation, the $m t s^{X E 2258}$, ina $C^{P 209} /$ ina $C^{P 209}$ double mutant displays almost wild-type deactivation kinetics.

\section{A reduction of $\mathrm{PP} 2 \mathrm{~A}$ fails to rescue the abnormal light} adaptation of ina $^{P 209}$ heterozygotes and homozygotes The ina $C^{P 209}$ homozygotes were reported to exhibit defective light adaptation, a feedback mechanism that permits photoreceptors to adjust their light sensitivity over a wide range of ambient illumination. Therefore, in the presence of the background light, photoreceptors show a reduced response, compared with with- 
A

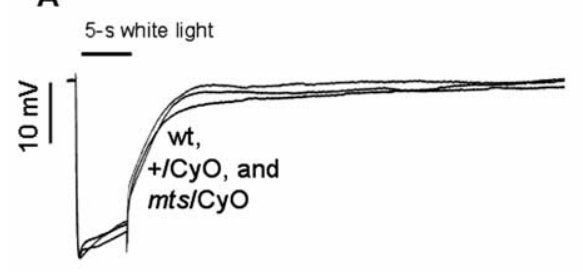

B
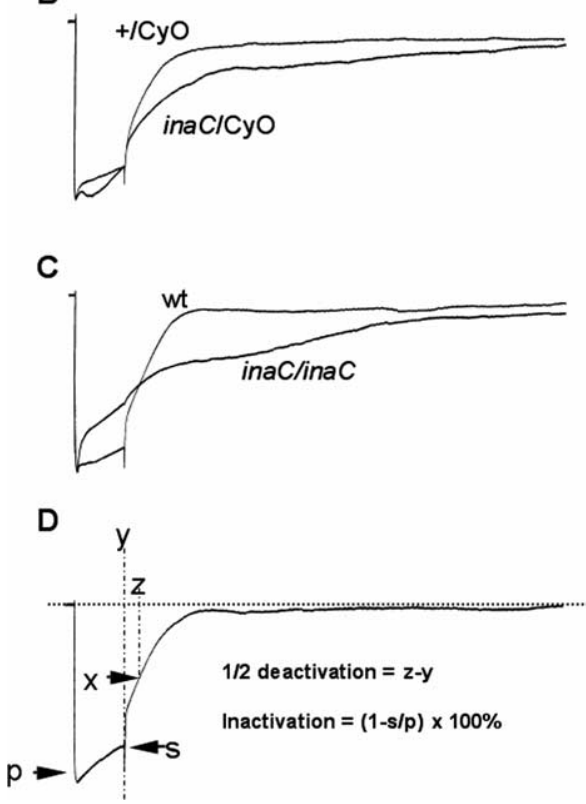

E

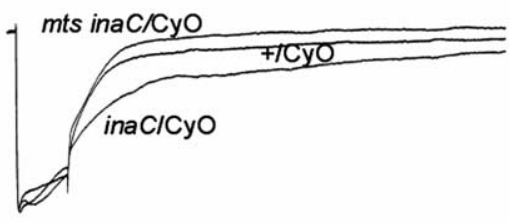

$\mathbf{F}$

G
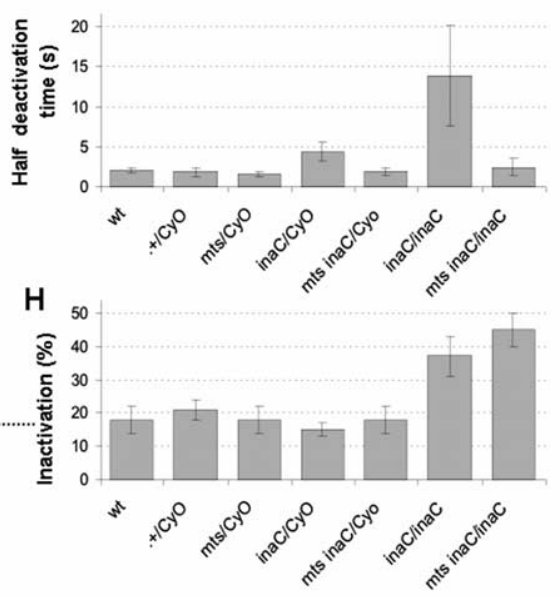

Figure 5. Electroretinogram analyses of wild-type and mutant flies affecting $m t^{X E 2258}{ }^{\text {, inac }} C^{P 209}$ or double mutants, in response to a $5 \mathrm{~s}$ white light $\left(\log I / I_{0}=-1\right)$. $\boldsymbol{A}$, The $m t s^{X E 2258}$ heterozygote displays wild-type ERG response. Shown are superimposed ERG tracings of wild type (wt or $+/ \mathrm{Cy} 0)$ and the $m t^{X E 2258}$ heterozygote $(m t s / C y 0)$. $\boldsymbol{B}$. The inac ${ }^{P 209}$ is semidominant, because the ina $C^{P 209}$ heterozygote (inaC/Cy0) exhibits slow deactivation. $C$, The ina $C^{P 209}$ homozygote displays response inactivation and slow deactivation. $\boldsymbol{D}, \mathrm{A}$ diagram highlighting the parameters for defining the half deactivation time and relative inactivation of the ERG response. The half deactivation time is the elapsed time between $y$ and $z$. Inactivation is defined as (1 $s / p) \times 100 \%$, where $p$ corresponds to the initial peak amplitude and $s$ corresponds to the amplitude at the end of $5 s$ pulse. $\boldsymbol{E}$, The $m t^{X E 2258}$ heterozygote rescues the deactivation defects of ina ${ }^{P 209}$ heterozygotes. Shown are three superimposed ERG responses corresponding to $\mathrm{mts}^{X E 2258}$, ina $\mathrm{C}^{P 209}$ double heterozygotes $\left(\mathrm{mts}\right.$, inaC/CyO), ina $\mathrm{C}^{P 209}$ heterozygotes, and wild-type flies. $\boldsymbol{F}$, The $m t^{X E 2258}$ heterozygote fails to rescue the response inactivation but modifies the slow deactivation of ina ${ }^{P 209}$ homozygotes. G, A comparison of the half deactivation time of various mutants. Shown is a histogram depicting the half deactivation time (mean \pm $\mathrm{SD} ; n=7)$. $\boldsymbol{H}$, A comparison of the relative inactivation (mean $\pm \mathrm{SD} ; n=7$ ) in wild type and mutants. Abbreviations: $m t s$, mts $^{\text {XE2258; inaC, inaC }}{ }^{P 209}$.

out background light. We questioned whether ina $C^{P 209}$ heterozygotes have adaptation defects, and if so, whether $m t s^{X E 2258}$ alters this abnormal visual electrophysiology. To characterize the light adaptation, we used an established protocol by measuring the response to a $5 \mathrm{~s}$ test light pulse $\left(\log I / I_{\mathrm{o}}=-1\right)$, with a continuous background light of $35 \mathrm{~s}$ as depicted in Figure $6 \mathrm{~A}$. For each background intensity, the response to the test pulse is denoted as an arrow that corresponds to the peak amplitude. The peak amplitude is normalized to that obtained without background illumination, and plotted against the background intensity as shown in Figure $6 B$. We demonstrate that both ina $C^{P 209}$ homozygotes and heterozygotes display a similar degree of deficiency in light adaptation, as the response curve shifted to the right, and this shift is very significant in dim background light. This shift indicates that the ina $C^{P 209}$ mutants exhibit a greater response as they were less adapted to dim light. Importantly, both ina $C^{\text {P209 }}$ mts ${ }^{\text {XE2258 }}$ double heterozygotes and $m t^{X E 2258}$, ina $C^{P 209} /$ ina $^{\text {P209 }}$ double mutants also display comparable light adaptation defects, similar to ina $C^{P 209}$ mutants. This result indicates that $m t s^{X E 2258}$ fails to correct the adaptation deficits in both ina $C^{P 209}$ heterozygotes and homozygotes. Our findings further support the haploinsufficient nature of the inaC mutation to bring about light adaptation. We conclude that PP2A does not play a role to modulate the eye-PKC-dependent light adaptation process.

\section{PP2A does not modify the activity of NORPA}

PP2A only rescues one of the three ina $C^{P 209}$ defects, suggesting that it works downstream of eye-PKC. To investigate whether PP2A also plays a role modulating the upstream events of eye-PKC, we examined whether MTS affects NORPA, a PLC $\beta$ that catalyzes the breakdown of $\mathrm{PIP}_{2}$ (phosphatidylinositol-4,5-bisphosphate) to release second messengers, by double mutant analysis. We chose norpA $A^{P 16}$, a hypomorphic allele with a partially active PLC $\beta$, which displays ERG with extremely slow deactivation kinetics (Fig. 6C) (Yoon et al., 2004). We show that double mutants (norpA; $m t s / \mathrm{CyO}$ ) exhibit ERG waveform similar to that of norp $A^{P 16}$, with the characteristic deactivation kinetics (Fig. 6C). This finding indicates that a reduction of the PP2A activity fails to rescue the slow deactivation of norp $A^{P 16}$, supporting that PP2A is not involved in regulating NORPA in the visual cascade.

\section{Discussion}

Here, we present the results from several distinct biochemical and electrophysiological assays, each shedding light on the key role that PP2A plays in Drosophila visual transduction. Our in vitro assays show that PP2A dephosphorylates the scaffolding protein INAD, opposing the activity of eye-PKC to phosphorylate INAD. We also show an increased level of INAD phosphorylation in three distinct $m t s$ heterozygotes, wherein the catalytic $\mathrm{C}$ subunit of PP2A has been rendered ineffective. Utilizing ERG recordings, in partial loss-of-function mutants of $m t s^{X E 2258}$, ina $C^{P 209}$, and the combined double mutant, we found that PP2A and eye-PKC have opposing physiological functions, and that a balance between the activities of eyePKC and PP2A is central for the proper deactivation of the visual response. Our results strongly indicate that PP2A appears to impact signaling proteins operating downstream of NORPA in the visual cascade. Integrating our in vivo and in vitro findings into the current model of eye-PKC-mediated regulation of INAD, we conclude that reversible phosphorylation of INAD is dependent on the opposing enzymatic actions of eye-PKC and PP2A and that phosphorylation of INAD is critical for fast deactivation of the visual signaling process.

The multiple biochemical assays that we conducted strongly support PP2A as a key phosphatase responsible for the dephosphorylation of INAD. Based on both its inhibition profile with 
okadaic acid, and its copurification alongside the FPLC fraction with positive phosphatase activity, we identified PP2A as the prime candidate for mediating INAD dephosphorylation. After finding that a purified A/C dimer of PP2A dephosphorylates INAD in vitro, we followed up performing immunocomplex kinase assays. As expected, a reduction in PP2A catalytic efficiency causes a significant increase in the measurable fraction of phosphorylated INAD. By examining three distinct $m t s$ heterozygotes, each carrying a $\mathrm{C}$ subunit mutation resulting in a partial loss of PP2A function, we demonstrate a significant increase in INAD phosphorylation levels with $m t s^{X E 2258}$ exhibiting the most dramatic increase. The enhanced INAD phosphorylation observed in the $m t s$ extracts strongly suggests that PP2A is closely positioned in the INAD complex to promote timely dephosphorylation of INAD. Consistently, we demonstrate that PP2A can be coisolated with INAD, thus representing a newly identified component of the INAD macromolecular complex.

For insights into the role PP2A plays in Drosophila vision, we studied $m t s^{X E 2258}$ heterozygotes and found a surprisingly normal ERG waveform. Although a reduction in the level of active PP2A in vivo has no effect on visual function, we found that $i_{n a} C^{P 209}$ heterozygotes exhibit abnormal light adaptation, as well as delayed deactivation of visual signaling. ina $C^{P 209}$ and $m t s^{X E 2258}$ both encode for enzymes; why would missing one functional copy of the PP2A gene not affect normal visual electrophysiology, whereas missing a functional copy of the eye-PKC gene drastically slows deactivation? ina $C^{P 209}$ must be a semidominant mutation; in other words, inaC is haploinsufficient. An explanation for the haploinsufficiency of the eye-PKC gene is that the substrate repertoire of eye-PKC is defined by substrate colocalization to the INAD macromolecular complex to which eye-PKC is tethered. This hypothesis is in good agreement with our previous observation that the interaction with INAD is essential for the in vivo function of eye-PKC to modulate the visual response (Adamski et al., 1998).

Although MTS is also tethered to the INAD signaling complex, unlike eye-PKC, the abundance of MTS relative to INAD in photoreceptors is likely to make it less sensitive to a reduction of its gene dosage to effect visual electrophysiology. Alternatively, it is possible that anchoring to the INAD complex by PP2A may be regulated by the interaction via its $B$ subunit instead of the $C$ subunit. Therefore, a reduction of MTS may not significantly modify its presence in the INAD complex.

To elucidate the functional interplay, in vivo, between eyePKC and MTS, we characterized $m t s^{X E 2258}$ and inaC ${ }^{P 209}$ double heterozygotes. We made a significant discovery that only the slow deactivation defect was rescued in the $m t s^{X E 2258}$ heterozygous background. The selective rescue of deactivation defects by $m t s^{\text {XE2258 }}$, with no rescue of the abnormal light adaptation found in ina $\mathrm{C}^{P 209}$ heterozygotes, suggests that PP2A regulates proteins that lie downstream of eye-PKC, a conclusion that is also sup-
C
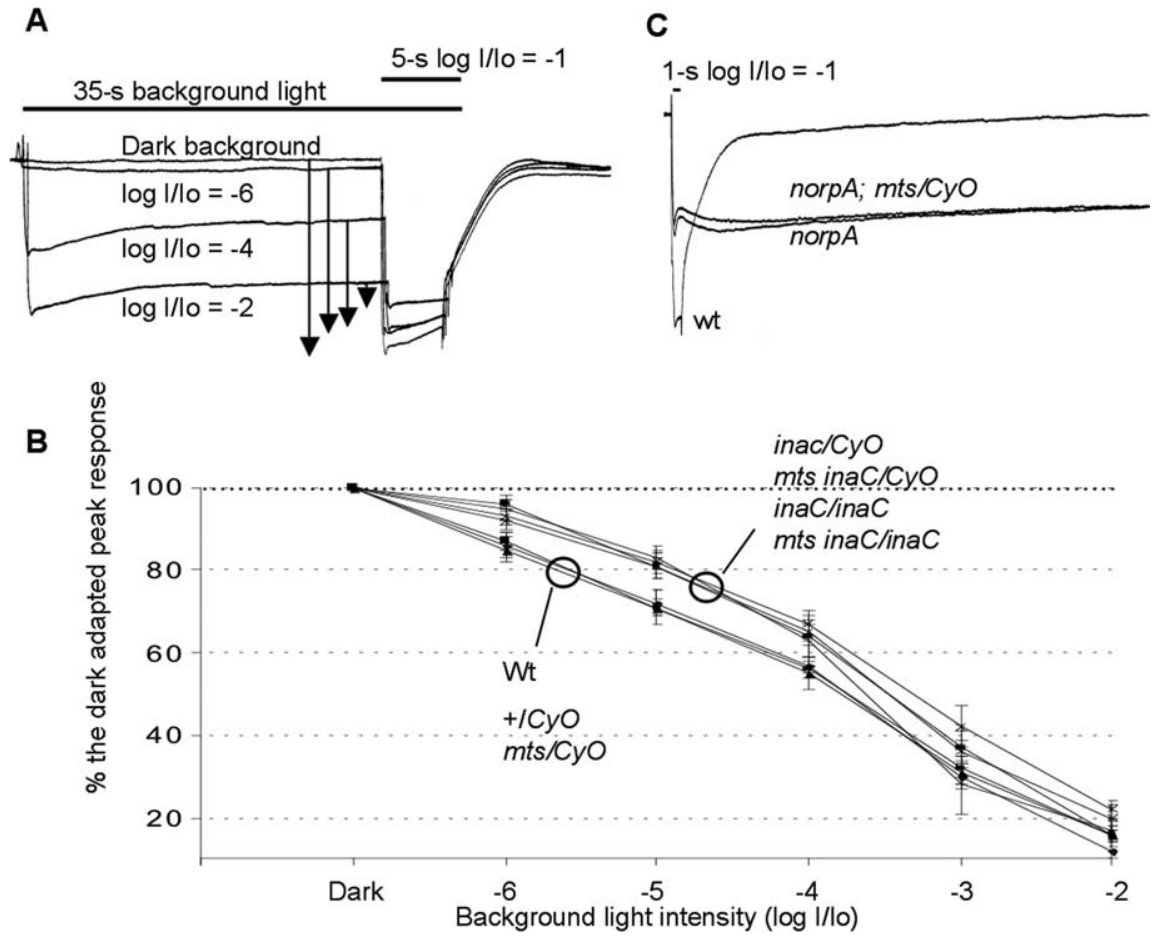

Figure 6. $m t^{X E 2258}$ fails to rescue the light adaptation defects of ina ${ }^{P 209}$ heterozygote and homozygote, and the slow .

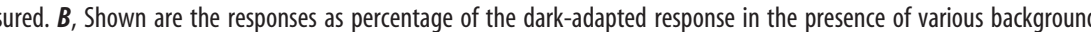
illuminations. C, Both norpA ${ }^{P 16}$ and $m t^{X E 2258}$, norp $A^{P 16}$ double mutants display similar slow deactivation kinetics. Abbreviations: $m t s, m^{X E 2258}$; inaC, ina ${ }^{\text {p209; }}$; norpA, norpA ${ }^{\text {P16. }}$.

ported by the inability of $m t s^{X E 2258}$ to restore the slow deactivation defect in a hypomorphic allele of norp ${ }^{P 16}$.

A concomitant reduction of the PP2A activity would lead to increased phosphorylation of multiple substrates contributing to the observed normal, fast deactivation kinetics found in the double mutant. Potential PP2A substrates may include INAD, TRP, and eye-PKC. Like other conventional PKCs, eye-PKC is most likely a phosphoprotein (Newton, 2003) and hence its catalytic activity is sensitive to PP2A. It is expected that a reduction of PP2A should increase the autophosphorylation of eye-PKC, bringing about an enhanced catalytic capability. However, the presumably increased eye-PKC activity fails to restore the light adaptation abnormality, suggesting that the modulation of eyePKC represents a lesser role of PP2A in vivo.

It is possible that PP2A dephosphorylates TRP, thus regulating deactivation kinetics. Consistently, we have reported that a lack of phosphorylation at Ser ${ }^{982}$ of TRP leads to slowed deactivation of the visual response (Popescu et al., 2006). However, $\operatorname{trp}{ }^{P 343}$, a null allele affecting the $\operatorname{trp}$ gene, displays a less severe defect in deactivation than that of ina $C^{P 209}$ (H.-T. Leung and W. L. Pak, unpublished observations), suggesting eye-PKC phosphorylation of TRP does not play a major role in the normal deactivation. We have begun to examine the eye-PKC-dependent phosphorylation of INAD and our preliminary results suggest that a loss of phosphorylation in INAD also results in slowed deactivation kinetics (N. Wang and B.-H. Shieh, unpublished observations). This finding together with our aforementioned biochemical results supports our hypothesis that the phosphatase PP2A directly regulates phosphorylation states of INAD to impact fast deactivation of the visual signaling. Very recently, Mishra et al. (2007) reported a light-dependent conformation 
change in the fifth postsynaptic density-95/Discs large/zona occludens-1 (PDZ) domain of INAD and proposed that eye-PKC might orchestrate this event. Their findings are in agreement with our studies supporting a critical role of INAD phosphorylation to promote fast deactivation. It will be of great interest to elucidate how phosphorylation of INAD leads to the conformation switch in its fifth PDZ domain.

In addition to INAD, PP2A may dephosphorylate other, yetto-be identified substrates. Our observations that $m t s^{X E 2258}$ modified the severe deactivation defect of ina $C^{P 209}$ homozygotes, suggests that a reduction of MTS increases phosphorylation of proteins, which are regulated by non-eye-PKC serine/threonine protein kinases. Several protein kinases including CaMKII (Kahn and Matsumoto, 1997), and NINAC (neither inactivation nor afterpotential C) (Montell and Rubin, 1988; Li et al., 1998) have been shown to modulate deactivation, and may play a role in regulating deactivation in the absence of eye-PKC.

In summary, here we demonstrate the roles of PP2A and eye$\mathrm{PKC}$ in orchestrating reversible phosphorylation of INAD, and that phosphorylation of INAD is most likely involved in fast deactivation kinetics of the visual signaling in Drosophila. Our multiple biochemical findings support the critical role of PP2A to dephosphorylate INAD. Our electrophysiological characterization strongly indicates that a reduction of PP2A compensate for a partial loss of function in eye-PKC leading to rescuing the slow deactivation defect.

\section{References}

Adamski FM, Zhu MY, Bahiraei F, Shieh BH (1998) Interaction of eye protein kinase C and INAD in Drosophila. Localization of binding domains and electrophysiological characterization of a loss of association in transgenic flies. J Biol Chem 273:17713-17719.

Barford D (1996) Molecular mechanisms of the protein serine/threonine phosphatases. Trends Biochem Sci 21:407-412.

Bloomquist BT, Shortridge RD, Schneuwly S, Perdew M, Montell C, Steller H, Rubin G, Pak WL (1988) Isolation of a putative phospholipase C gene of Drosophila, norpA, and its role in phototransduction. Cell 54:723-733.

Gu Y, Oberwinkler J, Postma M, Hardie RC (2005) Mechanisms of light adaptation in Drosophila photoreceptors. Curr Biol 15:1228-1234.

Hardie RC (2004) Regulation of Drosophila TRP channels by lipid messengers. Novartis Found Symp 258:160-171,263-266.

Hardie RC, Peretz A, Suss-Toby E, Rom-Glas A, Bishop SA, Selinger Z, Minke B (1993) Protein kinase C is required for light adaptation in Drosophila photoreceptors. Nature 363:634-637.

Huber A, Sander P, Paulsen R (1996a) Phosphorylation of the InaD gene product, a photoreceptor membrane protein required for recovery of visual excitation. J Biol Chem 271:11710-11717.

Huber A, Sander P, Gobert A, Bahner M, Hermann R, Paulsen R (1996b) The transient receptor potential protein (Trp), a putative store-operated $\mathrm{Ca}^{2+}$ channel essential for phosphoinositide-mediated photoreception, forms a signaling complex with NorpA, InaC and InaD. EMBO J 15:7036-7045.

Huber A, Sander P, Bahner M, Paulsen R (1998) The TRP $\mathrm{Ca}^{2+}$ channel assembled in a signaling complex by the PDZ domain protein INAD is phosphorylated through the interaction with protein kinase $\mathrm{C}$ (ePKC). FEBS Lett 425:317-322.

Kahn ES, Matsumoto H (1997) Calcium/calmodulin-dependent kinase II phosphorylates Drosophila visual arrestin. J Neurochem 68:169-175.

Kloeker S, Wadzinski BE (1999) Purification and identification of a novel subunit of protein serine/threonine phosphatase 4. J Biol Chem 274:5339-5347.

Larrivee DC, Conrad SK, Stephenson RS, Pak WL (1981) Mutation that selectively affects rhodopsin concentration in the peripheral photoreceptors of Drosophila melanogaster. J Gen Physiol 78:531-545.

Lechward K, Awotunde OS, Swiatek W, Muszynska G (2001) Protein phosphatase 2A: variety of forms and diversity of functions. Acta Biochim Pol 48:921-933.
Lee YJ, Dobbs MB, Verardi ML, Hyde DR (1990) dgq: a Drosophila gene encoding a visual system-specific $\mathrm{G}$ alpha molecule. Neuron 5:889-898.

Li HS, Montell C (2000) TRP and the PDZ protein, INAD, form the core complex required for retention of the signalplex in Drosophila photoreceptor cells. J Cell Biol 150:1411-1422.

Li HS, Porter JA, Montell C (1998) Requirement for the NINAC kinase/ myosin for stable termination of the visual cascade. J Neurosci 18:9601-9606.

Liu M, Parker LL, Wadzinski BE, Shieh BH (2000) Reversible phosphorylation of the signal transduction complex in Drosophila photoreceptors. J Biol Chem 275:12194-12199.

Lu CS, Hodge JJ, Mehren J, Sun XX, Griffith LC (2003) Regulation of the $\mathrm{Ca}^{2+} / \mathrm{CaM}$-responsive pool of CaMKII by scaffold-dependent autophosphorylation. Neuron 40:1185-1197.

Mishra P, Socolich M, Wall MA, Graves J, Wang Z, Ranganathan R (2007) Dynamic scaffolding in a $G$ protein-coupled signaling system. Cell 131:80-92.

Montell C, Rubin GM (1988) The Drosophila ninaC locus encodes two photoreceptor cell specific proteins with domains homologous to protein kinases and the myosin heavy chain head. Cell 52:757-772.

Moses K, Ellis MC, Rubin GM (1989) The glass gene encodes a zinc-finger protein required by Drosophila photoreceptor cells. Nature 340:531-536.

Newton AC (2003) Regulation of the ABC kinases by phosphorylation: protein kinase $\mathrm{C}$ as a paradigm. Biochem J 370:361-371.

Pak WL, Leung HT (2003) Genetic approaches to visual transduction in Drosophila melanogaster. Receptors Channels 9:149-167.

Popescu DC, Ham AJ, Shieh BH (2006) Scaffolding protein INAD regulates deactivation of vision by promoting phosphorylation of transient receptor potential by eye protein kinase C in Drosophila. J Neurosci 26:8570-8577.

Ranganathan R, Harris GL, Stevens CF, Zuker CS (1991) A Drosophila mutant defective in extracellular calcium-dependent photoreceptor deactivation and rapid desensitization. Nature 354:230-232.

Sathyanarayanan S, Zheng X, Xiao R, Sehgal A (2004) Posttranslational regulation of Drosophila PERIOD protein by protein phosphatase 2A. Cell 116:603-615.

Scott K, Becker A, Sun Y, Hardy R, Zuker C (1995) Gq alpha protein function in vivo: genetic dissection of its role in photoreceptor cell physiology. Neuron 15:919-927.

Shieh BH, Niemeyer B (1995) A novel protein encoded by the InaD gene regulates recovery of visual transduction in Drosophila. Neuron 14:201-210.

Shieh BH, Zhu MY (1996) Regulation of the TRP $\mathrm{Ca}^{2+}$ channel by INAD in Drosophila photoreceptors. Neuron 16:991-998.

Smith DP, Ranganathan R, Hardy RW, Marx J, Tsuchida T, Zuker CS (1991) Photoreceptor deactivation and retinal degeneration mediated by a photoreceptor-specific protein kinase C. Science 254:1478-1484.

Snaith HA, Armstrong CG, Guo Y, Kaiser K, Cohen PT (1996) Deficiency of protein phosphatase $2 \mathrm{~A}$ uncouples the nuclear and centrosome cycles and prevents attachment of microtubules to the kinetochore in Drosophila microtubule star (mts) embryos. J Cell Sci 109:3001-3012.

Steele FR, Washburn T, Rieger R, O’Tousa JE (1992) Drosophila retinal degeneration $\mathrm{C}(\mathrm{rdgC})$ encodes a novel serine/threonine protein phosphatase. Cell 69:669-676.

Tsunoda S, Sierralta J, Sun Y, Bodner R, Suzuki E, Becker A, Socolich M, Zuker CS (1997) A multivalent PDZ-domain protein assembles signalling complexes in a G-protein-coupled cascade. Nature 388:243-249.

Wang T, Montell C (2007) Phototransduction and retinal degeneration in Drosophila. Pflügers Arch 454:821-847.

Wassarman DA, Solomon NM, Chang HC, Karim FD, Therrien M, Rubin GM (1996) Protein phosphatase 2A positively and negatively regulates Ras1-mediated photoreceptor development in Drosophila. Genes Dev 10:272-278.

Yates III JR, Eng JK, McConnack AL, Schieltz D (1995) Method to correlate tandem mass spectra of modified peptides to amino acid sequences in the protein database. Anal Chem 67:1426-1436.

Yoon J, Leung HT, Lee S, Geng C, Kim Y, Baek K, Pak WL (2004) Specific molecular alterations in the norpA-encoded phospholipase C of Drosophila and their effects on electrophysiological responses in vivo. J Neurochem 89:998-1008. 